\title{
Design and Implementation of Mobile Phone System for Estimating Nutrient Content of Foods
}

\author{
Xin Zheng ${ }^{1, a}$, Run Yao ${ }^{1, b}$, Yifei Gong ${ }^{1, c}$, Qinyi Lei ${ }^{1, d}$ and Qian Yin ${ }^{1, e^{*}}$ \\ ${ }^{1}$ Beijing Normal University, Beijing, China

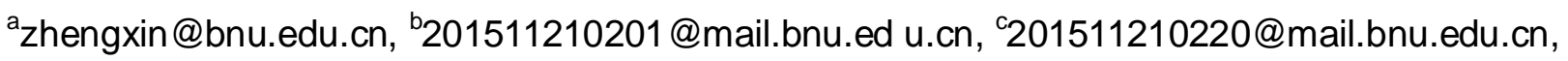 \\ d201511210126@mail.bnu.edu.cn, evinqian@bnu.edu.cn
}

Keywords: Food nutrition estimation; Mobile application; Dietary management

\begin{abstract}
With the increasing number of obese or overweight people, healthy diet has become a hot topic. Most of the health management software on the market lacks the function of estimating food nutrition, which causes users to be unable to accurately understand their own diet. Based on the food volume calculation method, a mobile phone estimating system that uses the combination of Client-Server and database to estimate food nutrition is designed and realized in this paper. It provides personalized dietary and nearby restaurants recommendations based on daily meals and physical indicators, which not only greatly increased the accuracy of food nutrition estimation, but also is convenient and quick.
\end{abstract}

\section{Introduction}

In 2014, "THE LANCET" reported that the number and proportion of obese and overweight people in the world had been increasing in the last 30 years. Global obesity and overweight population increased from 857 million in 1980 to 2.1 billion in 2013. [1].

Research shows that reasonable diet is an essential part of every traditional weight loss method, so accurately calculating the daily calories are crucial to weight loss or maintenance.

However, the existing diet health management software function is not perfect. Manika Puri[2] proposed a system based on point cloud for $3 \mathrm{~d}$ reconstruction of food, which is complicated. Rana al-maghrabi proposed another system [3], whose accuracy was greatly influenced by the reference frame.

In this paper, the volume of food was calculated by images of food and hand images, which is based on image segmentation and simple models nested volume estimation. The user selects the food type independently, and the nutritional content of the food is calculated. Combining daily meals and users' own body indicators, personalized diet recommendations are given. Compared with the traditional method, the calculation accuracy of the food nutrition composition is greatly improved.

\section{System Design}

The overall system architecture is shown in Fig. 1, using the combination of Client-Server and database. Its main modules include the databases, image analysis and processing modules, component calculation modules, information recommendation modules, and user interaction.

The databases that are established mainly include user's personal information; Simple three-dimensional model library; A database of food nutrients and the dietary recommendations database.

The image analysis processing module is based on the k-means image segmentation method. Then, after extracting the target object, the next module will be processed. 
Establish the component calculation module to ensure the accuracy of selected food. By analyzing data, according to database, the real-time analysis of images uploaded to users is realized.

Set up information recommendation, according to a database, in combination with users' preferences, personalized dietary recommendations are given. The overall operation mechanism of the system is shown in Fig. 2.

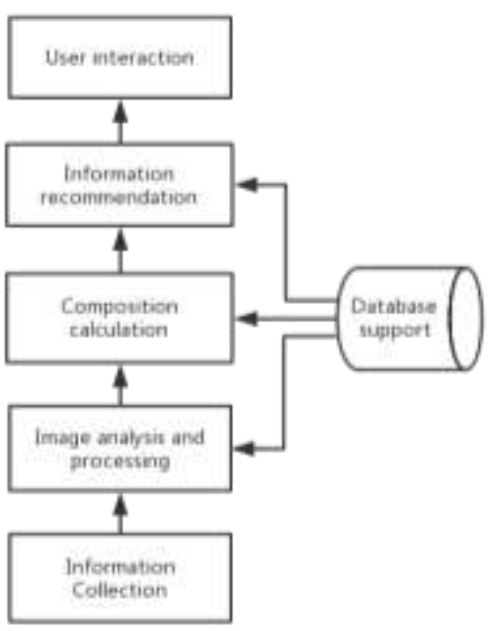

Figure 1. Overall system design

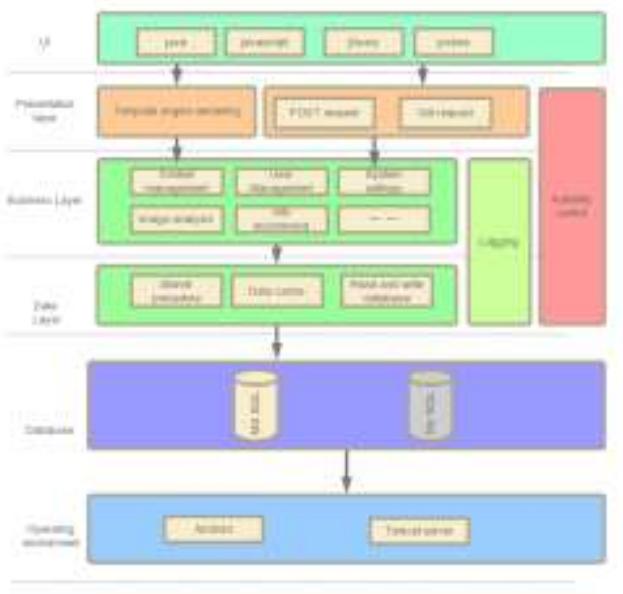

Figure 2. System operating mechanism

To sum up, the general process of users' use of the food evaluation system is shown in Fig. 3. First, we need pictures of food and hands on the same level from the mobile phone. We use the user's hand to estimate the volume. After we extract the target object, users will choose the food type.

After selecting the types, the size of the food is estimated by the user's palm. Compared with similar methods, ours is more practical. It is inconvenient that other methods need additional reference for calibration. we use a simple model nesting operation to calculate the food volume [4].

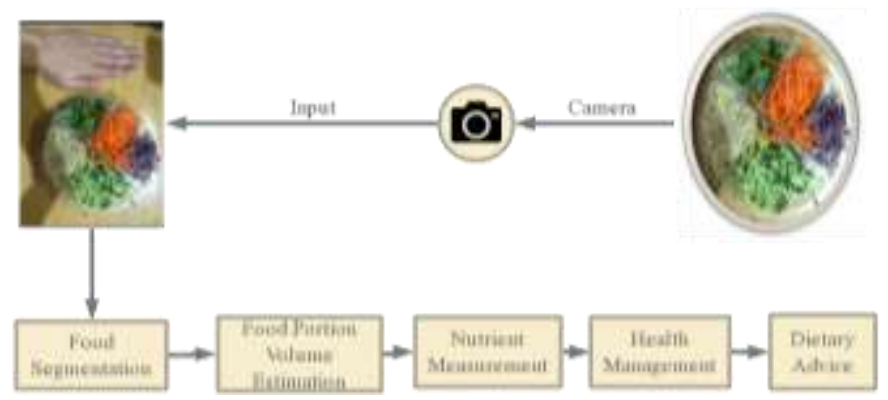

Figure 3. Overall system flow

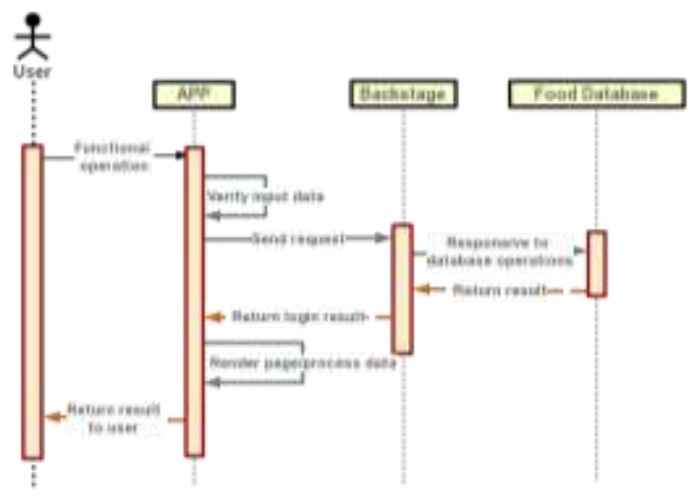

Figure 4. UML flowchart

Then, the system will calculate the nutrients, and present the results to the user. Finally, we will 
use personal information, combined with relevant nutrition knowledge, based on the user's collaborative filtering recommended personalized diet recommendations, ensure the overall diet structure. The overall UML sequence diagram of the project is shown in Fig. 4.

\section{Function Introduction of Each Module}

Food Nutrition Component Estimation Module. When users are registering, the basic indicators of the body and the length and width of the hand should be input. See Fig. 5(a) for the specific implementation.

The user needs to photograph three different angles of his hand and food at the same level, providing data information for subsequent food nutrient composition calculations, shown as Fig. $5(b)$.

Users need to manually select the type of food they eat according to the type of food provided by the interface. If there is no suitable food, add them manually, shown as Fig. 5(c).

Through the above steps, the project can calculate the food volume and corresponding nutrients at the backstage, then display the result to the user. See Fig. 5(d) for specific implementation

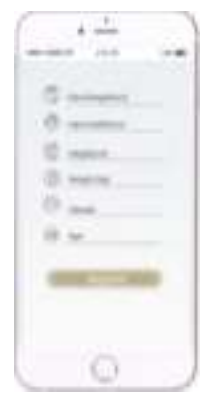

(a)

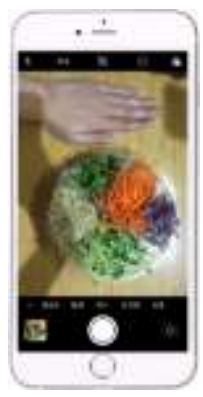

(b)

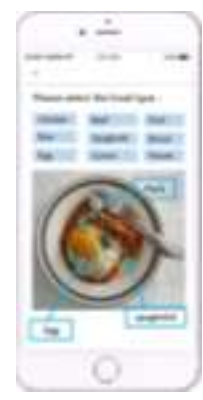

(c)

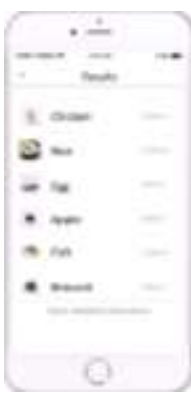

(d)

Figure 5. Food nutrition component estimation module (a) Information input interface (b) Food photography interface (c) Food selection interface (d) Nutrition composition estimation interface

Compared with the traditional same-type products, the realization of the function of this module can greatly improve the accuracy of food nutrition composition estimation, so as to make the users more effectively assess their own diet. This paper proposes to calculate the food volume by using the palm as the scale, because its accuracy and convenience.

Dietary Recommendation Module. Food recommendation. The APP this paper proposed calculate and analyze the user's diet, based on both food calories, sugar and other nutrients content related information and related database information diet. Besides, according to the daily calorie intake of Asians and their body's physical indicators, give the user a diet recommendation for the next meal. At the same time, the dishes with the same nutritional content will be selected based on users' preferences. The concrete implementation interface is shown in Fig. 6(a).

Restaurant recommendation. The APP also provides recommendations about nearby restaurants. All of these restaurants have recommended dishes, and also take into account geographic location, user preferences and spending power. The concrete implementation interface is shown in Fig. 6 (b). 


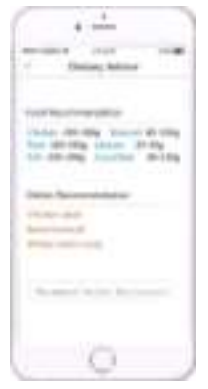

(a)

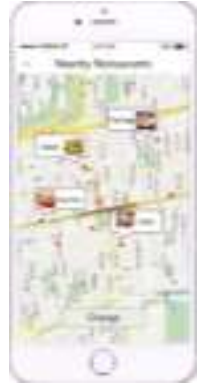

(b)

Figure 6. Dietary recommendations module. (a). Dietary recommendation (b). Nearby restaurants.

This paper proposed project will be based on the data of user's three meals per day, user's daily exercise data and its physical index, combining with the professional knowledge of nutrition for personalized dietary advice, to help different types of users more targeted to solve the problem, and have more reasonable diet. At the same time, the recommendation of the nearby restaurant can provide convenience for users, and it has a positive effect on the maintenance of reasonable diet. This function is seldom provided by the current catering mobile software, which is very innovative.

\section{Functional Realization and Advantages}

Food Nutrition Composition Estimation. The method of estimating food nutrition composition in this project is divided into four steps. The technical flow chart is shown in Fig. 7.

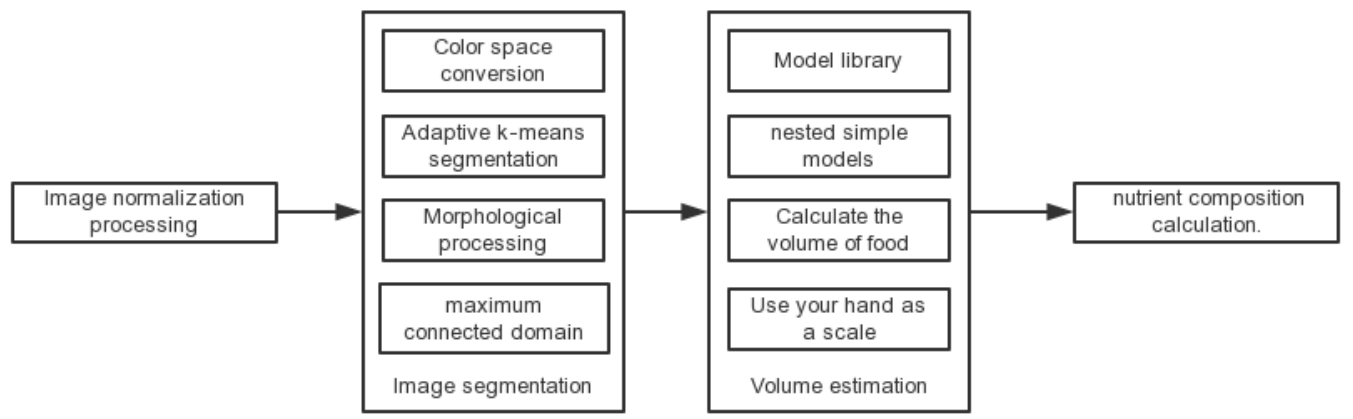

Figure 7. flow chat of food nutrition composition estimation

Because the image size is different, it need to be normalized.

Translate the picture into Lab color space and set the L component as a fixed value. The improved adaptive k-means algorithm is used to cluster the transformed images. After the value filtering in the gray image, the binary operation is performed, and the smaller noise in the image is partially filled, and the target object contour is extracted with the canny operator [5]. Finally, the maximal connected domain algorithm is used to get the segmentation result of the target image.

Establishes a $3 \mathrm{~d}$ model library containing the basic model, which includes the model name, shape, volume formula and parameter information. The food contour image was matched with the contour of the projection of the model, the one with the largest food matching degree was regarded as the model of the food. Take hand as reference, make sure the actual parameter of the model. The corresponding volume formula of the model is called to calculate the food volume.

After obtaining the food volume, the nutritional value and calorie index were estimated according to the different food density data obtained from the official website and the contents of various nutrients.

Food Recommendation. According to the user's ingestion history, the situation of body and the 
physical activity the user has taken, the project provides the user with a personal food recommendation from four steps as Fig. 8 shown.

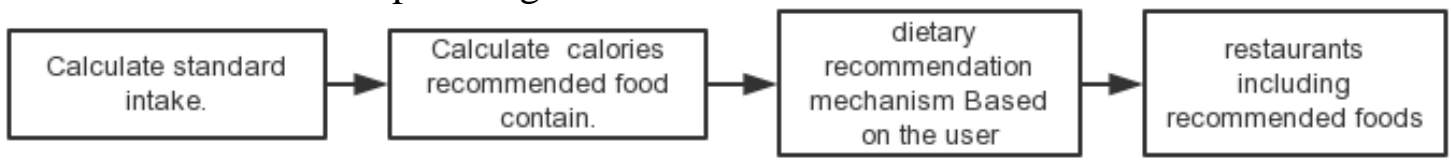

Figure 8. flow chat of food recommendation

According to the parameter of user's height, weight and so on to calculate the his standard value of eating. Then calculate the value of calories the recommended food should contain according to the daily history of food intake and the consumption of exercise.

Use dietary recommendation mechanism based on users. According to all users' preferences for items or information, find a "neighbor" user group similar to current user tastes and preferences. By using the algorithm of "k-nearest neighbor boor", based on the historical preference information of the $\mathrm{K}$ neighbors, the food whose calorie is up to standard will be recommended for user.

Use the peripheral search function. Configure the Android location SDK, get the map control, set the listening function, the restaurant containing the specified menu will be recommend to the user.

\section{Conclusion and Future Work}

In this article, we have constructed a healthy diet management APP for obese people, mainly based on image segmentation; according to hand scale, to obtain food volume. We present the nutrient content of the food to the user, and give the appropriate diet advice.

Compared with most of the existing diet analysis systems, our advantage lies in the creative use of hand as a scale for the calibration of the volume, which greatly facilitates the use. In addition, we have referenced a lot of nutrition-related knowledge to provide users with personalized and reasonable dietary advice, making dietary health management more user-friendly and intelligent.

In the future, we have the following research objectives:

We have some existing sports databases. In the future, we will improve and modify the sports database and integrate it into our system to recommend more comprehensive health advice for users.

Based on the dietary recommendations, we will continue to introduce relevant types of restaurant recommendations to provide users with more specific, time-saving and worry-free dietary advice.

We will continue to study and the currently covered dishes are still not comprehensive enough. In the future, we will conduct more relevant research on more extensive foods and dishes to cover more food types in order to expand the universality of the system and accommodate more people.

From the perspective of user experience, we will include community sharing features in our system. We intend to achieve the goal of building a healthy diet community by sharing information among users in the system, so as to better benefit more people.

\section{Acknowledgments}

The research work in this paper was supported by the grants from National Natural Science Foundation of China (61472043) and the Joint Research Fund in Astronomy (U1531242) under cooperative agreement between the NSFC and CAS. Prof. Qian Yin is the author to whom all the correspondence should be addressed. 


\section{Reference}

[1] THE LANCET (April 2018) on https://www.thelancet.com/

[2] M.Puri, Z.W. Zhu, Q. Yu, Recognition and Volume Estimation of Food Intake using a Mobile Device, J.IEEE Application of Computer Version,(2009)1-8

[3] R. Al-Maghrabi, Measuring Food Volume and Nutritional Values from Food Images,J.Menopause-the Journal of the North American Menopause Society,5(2013)3-10

[4] J. Dehais, M. Anthimopoulos, S. Shevchik et al, Two-View 3D Reconstruction for Food Volume Estimation,J.IEEE Transactions on Multimedia,19( 2017)1090-1099

[5] G.P. BIAN, Y.L. QIN, An adaptive edge-detection method based on Canny algorithm, J.Electronics and Technology, (2017)3-9 